\title{
Bate-papo Colaborativo ou Centrado no Professor?
}

\author{
Valleska C. M. da Silva ${ }^{1}$, Mariano Pimentel ${ }^{1}$, Vânia M. F. Dias ${ }^{1}$ \\ ${ }^{1}$ Programa de Pós-Graduação em Informática - Universidade Federal do Estado do Rio \\ de Janeiro (UNIRIO) - Rio de Janeiro - RJ - Brasil \\ \{valleska.dasilva, pimentel, vania\}@uniriotec.br
}

\begin{abstract}
Resumo. $O$ bate-papo, apesar de ser um meio de conversação em que se estabelece a relação todos-para-todos entre os interlocutores, na educação pode ocorrer do professor se tornar o centro das atenções aproximando-se de um modelo todos-um. Para caracterizar a relação estabelecida entre os sujeitos em uma sessão de bate-papo, a presente pesquisa propõe um método de análise com base nos conceitos de Análise de Redes Sociais para identificar se a conversação foi colaborativa (todos-todos) ou centrada no professor (todos-um). Isso nos faz questionar as práticas pedagógicas online que estão sendo realizadas por meio do bate-papo: o professor-mediador está promovendo a colaboração entre os alunos ou está reforçando a assimetria comunicacional entre os papéis professor-aluno tal como tipicamente ocorre na sala de aula presencial tradicional? Diferentemente do que se espera, muitas sessões de bate-papo são realizadas de modo centrado no professor, conforme discutido neste artigo.
\end{abstract}

Abstract. Chat is a conversation medium that establishes the many-to-many relationship between the interlocutors. However when used in the educational settings the teacher can become the center of the conversation, which characterizes the many-to-one relationship model. To characterize the relationship established during a chat session, this research proposes an analysis method based on Social Network Analysis to identify whether the conversation was collaborative (many-to-many) or centered on the teacher (many-to-one). The goal is to investigate whether the chat is being used to promote collaboration among students, or is further strengthening the asymmetry between teacher and students as typically occurs in a traditional classroom. Surprisingly, many chat sessions are centered on the teacher, as shown in this article.

\section{Introdução}

A educação a distância tem crescido muito, especialmente no Brasil: de praticamente zero curso no ano 2000, em uma década esta modalidade passou a ser responsável por quase $15 \%$ dos graduandos brasileiros, totalizando cerca de um milhão de estudantes nesta modalidade (INEP/MEC, 2012). Dentre os meios de interação, o bate-papo é um dos mais utilizados (junto com fórum e email), sendo adotado por mais de $60 \%$ das instituições brasileiras que ofertam cursos a distância com interação online (Censo EAD, 2012), o que evidencia a importância de pesquisas sobre o uso de bate-papo na educação.

Diversos autores reconhecem a adequação do bate-papo como meio para a realização de práticas pedagógicas (Silva, 2010; Stahl, 2009; Pimentel et al., 2003). O bate-papo possibilita a constituição de um espaço para realizar atividades diferentes da 
aula tradicional. A conversação informal, típica do bate-papo, possibilita o aluno perceber melhor o outro e perceber-se melhor como parte do grupo, proporciona um espaço para emoções que diminuem a sensação de isolamento tipicamente sentida na modalidade a distância. O uso contínuo do bate-papo integrado a atividades educacionais é uma forma de manter a motivação dos alunos e o engajamento para garantir o sucesso e a continuidade de um curso online.

O bate-papo estabelece a relação todos-todos entre os interlocutores (Calvão et al., 2014). Este modelo tem potencial para promover a colaboração entre os alunos, mas pode ocorrer do professor se tornar o centro das atenções no bate-papo reforçando a assimetria comunicacional estabelecida pelos papéis professor-aluno tal como tipicamente acontece na sala de aula presencial tradicional. Nesta pesquisa, busca-se caracterizar a relação que efetivamente se estabelece entre os participantes em uma sessão de bate-papo educacional, conforme discutido na Seção 2. Na Seção 3 são definidos os conceitos necessários para a compreensão da modelagem em grafo da troca de mensagens entre os interlocutores numa sessão de bate-papo, e as métricas de Análise de Redes Sociais (ARS) que adotamos. Além disso, ainda na Seção 3, propomos um algoritmo para caracterizar se a conversação estabelecida foi colaborativa ou centrada no professor. Na Seção 4, o algoritmo apresentado é usado para analisar $\log s$ reais de bate-papo ocorridos em contexto educacional. A conclusão desta pesquisa é apresentada na Seção 5.

\section{Bate-papo na Educação}

A prática pedagógica adotada na maioria das salas de aula (presenciais e online) ainda é baseada na cultura da transmissão de massa (modelo Um-Todos da Figura 1): o professor possui o conhecimento e transmite ao aluno, o aluno por sua vez recebe a informação de forma passiva.

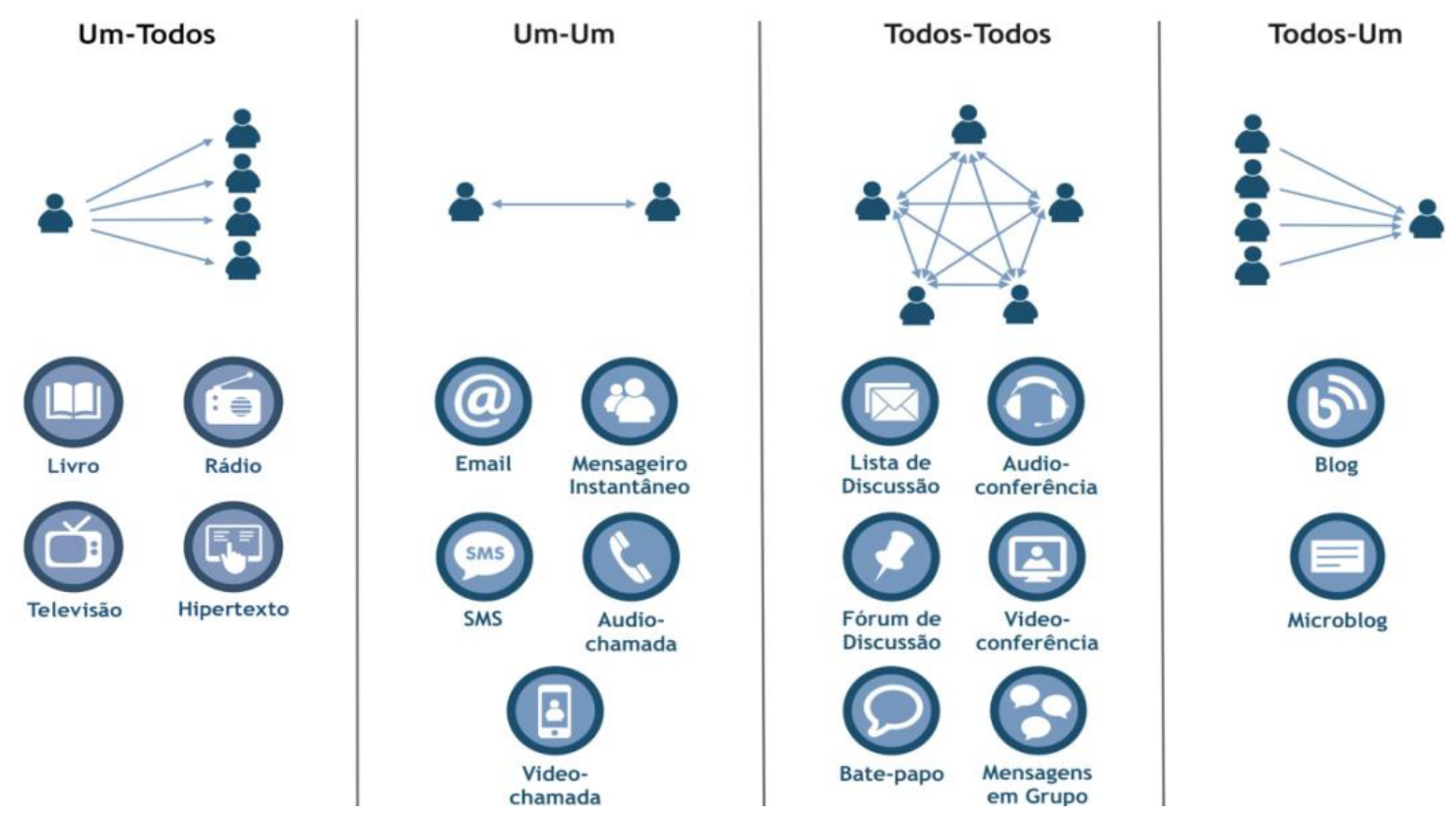

Figura 1. Relação entre os interlocutores nos diferentes meios de comunicação (baseado em Calvão et al., 2014)

Essa prática está ultrapassada em nossa sociedade contemporânea que é pósmassiva, cibercultural. Se antes as pessoas passavam horas na frente da TV como meros espectadores, hoje passam horas interagindo pelo computador. Os sujeitos praticam a 
discussão, a autoria, o compartilhamento, e evitam ambientes lineares que não possibilitam a participação. Diante dessa nova sociedade, não explorar devidamente a interação na educação é andar "na contramão da história" (Silva, 2010b).

A aprendizagem colaborativa é uma alternativa mais alinhada às necessidades da nova sociedade. A sala de aula colaborativa modifica os métodos tradicionais baseados na transmissão de conteúdos e promove a participação ativa e coletiva de todos mediados pelo professor. A ideia é retirar o foco sobre o ensino de conteúdos para enfocar a construção colaborativa do conhecimento pelos alunos com base em discussão, co-autoria, projetos em grupo, dentre outras práticas. A comunicação não deve ser Um-Todos, mas sim uma conversação Todos-Todos (Figura 1).

O bate-papo é reconhecido como um meio de conversação adequado para a realização de práticas pedagógicas colaborativas (Silva, 2010b; Pimentel et al., 2003). Pelo bate-papo, os interlocutores ficam conectados simultaneamente (comunicação síncrona) e trocam mensagens textuais geralmente curtas, sendo um meio voltado para a conversação em grupos pequenos e turmas com menos de 30 pessoas (Calvão et al., 2014; Rocha et al., 2015). O discurso no bate-papo se assemelha ao da conversação face-a-face, onde os usuários modificam a linguagem formal escrita para torná-la mais parecida com a linguagem oral, por exemplo, fazendo uso de onomatopeias e sobrecarga de pontuação. A rápida e intensa troca de mensagens e a informalidade do discurso reforçam a sensação de presença, o que possibilita o aluno perceber melhor o outro e perceber-se melhor como parte do grupo, proporcionando um espaço para emoções que diminuem a sensação de impessoalidade e isolamento.

Apesar de o bate-papo ser um meio em que se estabelece a relação Todos-Todos entre os interlocutores (pois qualquer um pode enviar uma mensagem, e as mensagens enviadas são distribuídas para todos), quando usado na educação a conversação pode ficar centrada no professor, conforme esquematizado na Figura 2. Ainda que ocorra a conversa entre os alunos, pode predominar a troca de mensagens dos alunos para o professor.

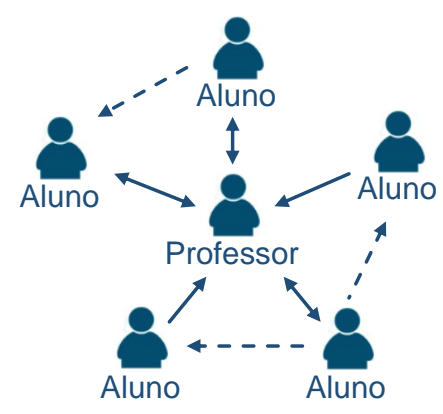

Figura 2. Conversação centrada no professor

Para caracterizar se a conversação no bate-papo foi colaborativa ou centrada no professor, é preciso analisar as associações entre as mensagens para identificar quem falou com quem. Alguns sistemas que implementam o bate-papo possibilitam "responder" uma mensagem, como exemplifica o sistema DebatePapo (Moraes, 2011; Netto, 2014), e neste caso fica registrado a relação entre a mensagem do participante B para o participante $A(B \rightarrow \mathbf{A})$. Alternativamente, a associação entre mensagens pode ser inferida pela análise do discurso. No presente artigo, foram usados tanto logs com associações inferidas por um analista do discurso quanto $\log s$ em que as associações foram estabelecidas diretamente no sistema pelos próprios participantes. Contabilizando 
a quantidade de mensagens trocadas entre os participantes da sessão, se for verificado uma predominância de mensagens destinadas ao professor, concluiremos que a conversação foi centrada no professor, caso contrário, concluiremos que a conversação foi colaborativa (distribuída). Este método é formalizado na seção a seguir.

\section{Algoritmo para Identificação de Conversa Centrada no Professor}

As redes sociais são comumente representadas por meio de grafos. Um grafo, $\mathbf{G}=(\mathbf{V}$, $\mathbf{E}$ ), consiste em um conjunto finito e não vazio $\mathbf{V}$ de elementos chamados vértices e em um conjunto finito $\mathbf{E}$ de pares de elementos distintos de $\mathbf{V}$, chamados arestas. Cada um dos pares de $\mathbf{E}$ pode ser ordenado, no caso de grafo direcionado, ou não, no caso de grafo simples. Um multigrafo é um grafo que pode possuir arestas múltiplas, ou seja, uma ou mais arestas entre o mesmo par de vértices. Arestas múltiplas podem ser substituídas por uma aresta única com peso, sem prejuízo do seu significado. Neste caso, o peso de uma aresta corresponde ao total de arestas substituídas. O grau de um vértice é igual ao número de arestas incidentes sobre ele. Seja $\mathbf{G}$ um grafo direcionado, então se define grau de saída como o número de arestas partindo de um vértice e grau de entrada, o número de arestas chegando a um vértice. Um grafo pode ser representado por uma matriz de adjacência $\mathbf{A}$ onde $\mathbf{A}_{\mathbf{i}, \mathbf{j}}$ representa o número de arestas entre os vértices $\mathbf{i} \mathbf{e} \mathbf{j}$, para $\mathbf{i}=\mathbf{1}$..n e $\mathbf{j}=\mathbf{1}$..n, onde $\mathbf{n}$ denota o número de vértices de $\mathbf{G}$. No presente trabalho, $\mathbf{A}_{\mathbf{i}, \mathbf{j}}$ é igual ao número de arestas partindo de $\mathbf{i}$ para $\mathbf{j}(\mathbf{i} \rightarrow \mathbf{j})$. Denominaremos A por matriz de adjacência ponderada. Usando a matriz de adjacência ponderada $\mathbf{A}$ de $\mathbf{G}$, o grau de saída $\mathbf{S}_{\mathbf{i}}$ de um vértice $\mathbf{i}$ pode ser obtido somando a linha $\mathbf{i}$ de $\mathbf{A}$, ao passo que o grau de entrada $\mathbf{T}_{\mathbf{j}}$ de $\mathbf{j}$ é obtido somando a coluna $\mathbf{j}$ de $\mathbf{A}$.

Utilizaremos o grafo direcionado para representar a conversação numa sessão de bate-papo, como exemplificado na Figura 3, em que os vértices representam os participantes da sessão e cada aresta e seu respectivo peso representam quantas mensagens A enviou para B. O grau de saída representa quantas mensagens o participante i enviou. $O$ grau de entrada representa quantas mensagens foram direcionadas para o participante $\mathbf{j}$.
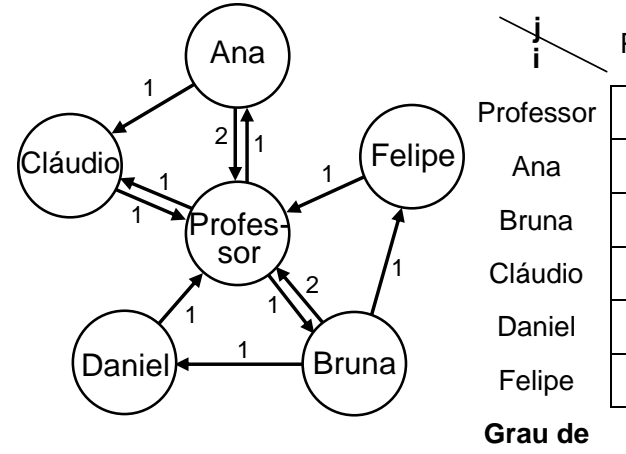

Entrada

\begin{tabular}{|c|c|c|} 
Professor & \multicolumn{1}{c}{ Ana } \\
\hline- & 1 & \\
\hline 2 & - & \\
\hline 2 & 0 & \\
\hline 1 & 0 & \\
\hline 1 & 0 & \\
\hline 1 & 0 & \\
\hline 7 & 1 \\
\hline
\end{tabular}

7 1

\begin{tabular}{l}
1 \\
\hline \\
\hline
\end{tabular}

Figura 3. Grafo e Matriz de Adjacência representando um cenário de troca de mensagens num bate-papo

A análise de redes sociais é o campo que estuda as relações sociais entre atores e os padrões e implicações dessas relações. Uma rede social pode ser abstraída como um grafo, onde os atores são representados por vértices e as relações entre os mesmos são expressas por arestas, tal como exemplificado na Figura $3 \mathrm{com}$ a modelagem da sessão de bate-papo por meio de um grafo direcionado sobre a troca de mensagens entre os participantes. É por meio da associação de cada mensagem (quem falou para quem) que mapeamos a rede social que se estabeleceu durante uma sessão de bate-papo. Estamos 


\section{CBIE-LACLO 2015}

Anais do XXVI Simpósio Brasileiro de Informática na Educação (SBIE 2015)

analisando, portanto, a rede social emergente de uma conversação (Recuero, 2012, p.143), especificamente da conversação realizada numa sessão de bate-papo.

Existem muitas métricas de centralidade para vértices individuais (Freeman, 2004). No contexto de bate-papo educacional, o número de participantes da sessão é reduzido (Calvão et al., 2014; Rocha et al., 2015) e assim muitas das métricas de centralidade não apresentam resultados significativos. Por esta razão, a métrica escolhida aqui foi a centralidade de grau, que é definida como o valor do grau de um vértice. Para este trabalho nos interessa em especial o grau de entrada, que indica a quantidade de mensagens recebidas por um participante (vértice). De acordo com Recuero (2012), além da importância do vértice, essa métrica pode indicar a popularidade e reputação de um determinado vértice na rede. Podemos ilustrar com o caso da Figura 3. Nessa figura, se nos concentrarmos no grau de saída, a aluna Bruna se destacará como uma participante central, pois foi a pessoa que mais enviou mensagens durante a sessão. Mas, apesar de ter falado bastante, Bruna não foi o foco de atenção daquela conversa, pois ao analisarmos o grau de entrada verificamos que Bruna está entre os que menos receberam mensagens, ou seja, ela não teve a atenção dos demais participantes. O foco da conversa era o professor, dado que para ele foram endereçadas muito mais mensagens do que para os demais participantes. Assim, o foco da conversação, neste contexto, é identificado através do grau de entrada e não pelo grau de saída.

Para analisar se a relação entre os interlocutores durante a sessão de bate-papo educacional foi centrada no professor, será utilizado o método de busca por Outlier traduzido como "ponto fora da curva" - através da realização do teste de diagrama de caixas. Um ponto fora da curva é um valor que apresenta um grande afastamento dos restantes, é um valor aberrante, anormal comparado com os demais valores do conjunto. No contexto do bate-papo educacional, um ponto fora da curva acima do limite superior pode indicar que um participante foi o foco da conversação obtendo muito mais atenção que os demais. O Teste de Diagrama de Caixas é capaz de identificar se, num conjunto de dados, existem ou não pontos fora da curva:

1. Calcula-se a mediana, o quartil inferior (Q1) e o quartil superior (Q3);

2. Calcula-se $\mathrm{IQR}=(\mathrm{Q} 3-\mathrm{Q} 1)$ e $\mathrm{Z}=1,5 \times \mathrm{IQR}$

3. São classificados como pontos fora da curva os valores inferiores a $(\mathrm{Q} 1-\mathrm{Z})$ e superiores a $(\mathrm{Q} 3+\mathrm{Z})$.

No método de análise que elaboramos nesta pesquisa, só nos interessam os pontos fora da curva superiores, visto que a centralidade da conversação é caracterizada pela discrepante quantidade de mensagens endereçadas a alguém. A fim de identificar se a conversação no bate-papo foi ou não centralizada, propomos o seguinte algoritmo:

Algoritmo AnalisarCentralidade

Entrada: $\log$ de bate-papo $\mathbf{G}=(\mathbf{V}, \mathbf{E})$

Saída: Decide se a sessão de bate-papo é ou não centrada

1. Obter a matriz de adjacência ponderada A de G;

2. Para cada $\mathrm{j} \in \mathrm{V}$, calcular o grau de entrada $\mathrm{Tj}$;

3. Ordenar o vetor $\mathrm{T}$ de modo crescente por valor do grau de entrada;

4. Aplicar o Teste de Diagrama de Caixas em T; 
5. Se houver ponto fora da curva acima do limite superior, retornar "conversação centrada", caso contrário retornar "conversação não centrada".

Caso a conversação seja centrada, o algoritmo descrito retorna quais são os pontos fora da curva, isto é, em qual ou quais pessoas esteve focada a conversação na sessão de bate-papo. Nesse caso, se o professor corresponde a um desses pontos então concluímos que ele manteve o papel de detentor da atenção. Por outro lado, se nenhum ponto fora da curva corresponde ao professor, concluímos que ele não dominou a conversa. Em ambos os casos de centralização, algumas medidas poderão ser tomadas pelo professor com o objetivo de incitar a maior participação por parte dos alunos.

Aplicando o algoritmo proposto no log de bate-papo exemplificado na Figura 3, obtemos o seguinte resultado:

1. A matriz de adjacência ponderada $A$ de $G$ está representada na Figura 3.

2. O grau de entrada está representado na Figura 3.

3. Vetor T ordenado: $\{1,1,1,1,2,7\}$

4. Teste de Diagrama de Caixas em T

$\bigcirc \quad$ Mediana $=1 ; \mathbf{Q 1}=1 ; \mathbf{Q 3}=1,75 ; \mathbf{I Q R}=0,75 ; \mathbf{Z}=1,125 ; \mathbf{Q 3}+\mathbf{Z}=2,875$

\section{Conversação centrada}

Portanto, seriam classificados como ponto fora da curva os valores superiores a 2,875. Nesse caso, o valor 7 é detectado como ponto fora da curva. Este é o valor do grau de entrada do professor. Assim, concluímos que a conversação nesta sessão de bate-papo ocorreu de forma centrada no professor.

\section{Análise dos dados}

Para ilustrar a aplicação do algoritmo aqui desenvolvido, apresentado na seção anterior, analisaremos três $\log s$ reais de bate-papo educacional nas subseções a seguir.

\subsection{Log WebDesign2013.2}

O $\log$ de bate-papo aqui analisado ocorreu na disciplina WebDesign de um curso de graduação em Sistemas de Informação de uma universidade federal. A sessão de batepapo foi realizada no Portal Tagarelas (Estruc, et al., 2012) e contava com oito participantes sendo sete alunos e um professor identificado pelo pseudônimo Paulo. Na Figura 4 são apresentadas a matriz adjacente e o grafo correspondente a este $\log$.

\begin{tabular}{|c|c|c|c|c|c|c|c|c|}
\hline & $\begin{array}{l}\frac{0}{2} \\
\text { ত }\end{array}$ & $\begin{array}{l}\text { टे } \\
\stackrel{0}{\frac{Z}{4}}\end{array}$ & $\begin{array}{l}\frac{\Phi}{\Phi} \\
\frac{D}{O} \\
\frac{D}{\Sigma}\end{array}$ & 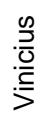 & $\begin{array}{l}\frac{\pi}{0} \\
\frac{\mathbb{0}}{\frac{0}{\sigma}} \\
\mathbb{\sigma}\end{array}$ & 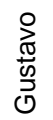 & $\begin{array}{l}\text { 음 } \\
\text { i } \\
i=\end{array}$ & 胥 \\
\hline Paulo & 0 & 2 & 7 & 2 & 1 & 4 & 2 & 6 \\
\hline Antony & 10 & 0 & 1 & 2 & 2 & 2 & 2 & 1 \\
\hline Michele & 7 & 0 & 0 & 0 & 1 & 3 & 0 & 3 \\
\hline Vinicius & 8 & 1 & 1 & 0 & 0 & 0 & 0 & 0 \\
\hline Gabriela & 13 & 3 & 2 & 1 & 0 & 1 & 1 & 1 \\
\hline Gustavo & 9 & 1 & 1 & 2 & 0 & 0 & 2 & 2 \\
\hline Tiago & 15 & 5 & 2 & 2 & 1 & 2 & 0 & 3 \\
\hline Anaí & 12 & 4 & 1 & 1 & 0 & 3 & 0 & 0 \\
\hline $\begin{array}{l}\text { Grau de } \\
\text { entrada }\end{array}$ & 74 & 16 & 15 & 10 & 5 & 15 & 7 & 16 \\
\hline
\end{tabular}

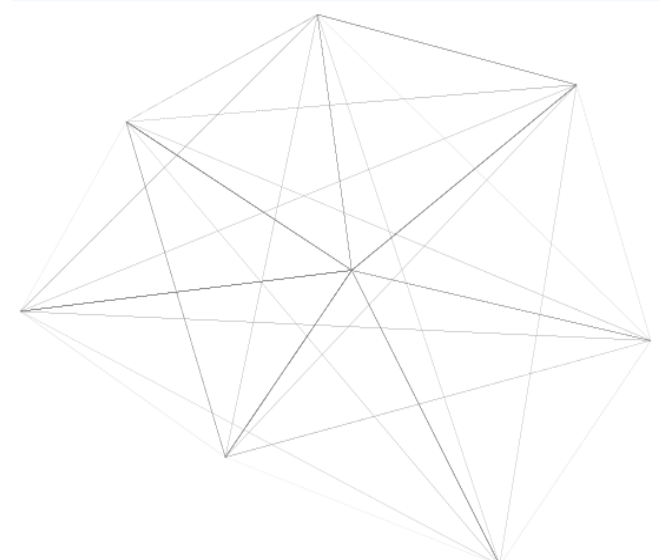

Figura 4. Matriz Adjacente e Grafo do Log WebDesign2013.2 


\section{CBIE-LACLO 2015}

Anais do XXVI Simpósio Brasileiro de Informática na Educação (SBIE 2015)

Aplicando o algoritmo na instância correspondente a este log, temos:

1. A matriz de adjacência ponderada $A$ de $\mathrm{G}$ está representada na Figura 4.

2. O grau de entrada está representado na Figura 4.

3. Vetor T ordenado: $\{5,7,10,15,15,16,16,74\}$

4. Teste de Diagrama de Caixas em T

○ Mediana=15; Q1=9,25; Q3=16; IQR=6,75; $\mathbf{Z}=10,125 ; \mathbf{Q 3}+\mathbf{Z}=26,125$

\section{Conversação centrada}

Logo, são classificados como ponto fora da curva os valores superiores a 26,125. De fato, é fácil identificar na matriz que o grau de entrada do professor, que foi endereçado em 74 mensagens, destoa dos demais valores. Ao professor foi endereçado um número discrepante de mensagens durante a sessão de bate-papo. $\mathrm{O}$ resultado da aplicação do algoritmo confirma essa observação, concluindo que essa sessão de batepapo foi centrada do professor.

\section{2. $\log$ SisColab2014.2}

Este $\log$ de bate-papo ocorreu na disciplina Sistemas Colaborativos do curso Mestrado em Informática de uma universidade federal. A sessão de bate-papo foi realizada com dez participantes sendo nove alunos e um professor identificado pelo pseudônimo Marcelo. A sessão também foi realizada no Portal Tagarelas. A Figura 5 mostra a matriz adjacente e o grafo correspondente a este log.

\begin{tabular}{|c|c|c|c|c|c|c|c|c|c|c|}
\hline & 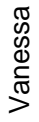 & $\begin{array}{l}\frac{0}{0} \\
\text { ग } \\
\frac{\sigma}{\Sigma}\end{array}$ & $\begin{array}{l}0 \\
0 \\
\frac{0}{\pi} \\
\Sigma\end{array}$ & 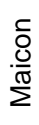 & $\frac{\frac{c}{\sigma}}{\bar{\alpha}}$ & $\frac{\frac{c}{0}}{\frac{0}{\bar{\sigma}}}$ & 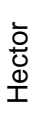 & $\frac{\widetilde{\sigma}}{\frac{\bar{\sigma}}{\widetilde{J}}}$ & $\begin{array}{l}\bar{\Phi} \\
\text { 유 } \\
\text { 으 }\end{array}$ & $\begin{array}{l}\frac{0}{\partial} \\
\frac{0}{\frac{0}{2}} \\
\frac{0}{1}\end{array}$ \\
\hline Vanessa & 0 & 10 & 1 & 3 & 1 & 2 & 0 & 0 & 0 & 0 \\
\hline Marcelo & 1 & 0 & 0 & 0 & 0 & 2 & 1 & 0 & 1 & 0 \\
\hline Marcos & 1 & 6 & 0 & 0 & 0 & 0 & 2 & 1 & 2 & 0 \\
\hline Maicon & 1 & 6 & 1 & 0 & 0 & 0 & 2 & 3 & 1 & 0 \\
\hline Allan & 0 & 5 & 2 & 0 & 0 & 0 & 0 & 0 & 0 & 0 \\
\hline Marlon & 0 & 1 & 2 & 0 & 1 & 0 & 1 & 0 & 2 & 0 \\
\hline Hector & 1 & 7 & 0 & 1 & 0 & 2 & 0 & 0 & 0 & 0 \\
\hline Camila & 1 & 7 & 1 & 0 & 0 & 1 & 2 & 0 & 2 & 1 \\
\hline Isabel & 0 & 7 & 4 & 1 & 0 & 0 & 1 & 1 & 0 & 0 \\
\hline Henrique & 0 & 6 & 0 & 0 & 0 & 0 & 0 & 0 & 0 & 0 \\
\hline $\begin{array}{l}\text { Grau de } \\
\text { entrada }\end{array}$ & 5 & 55 & 11 & 5 & 2 & 7 & 9 & 5 & 8 & 1 \\
\hline
\end{tabular}

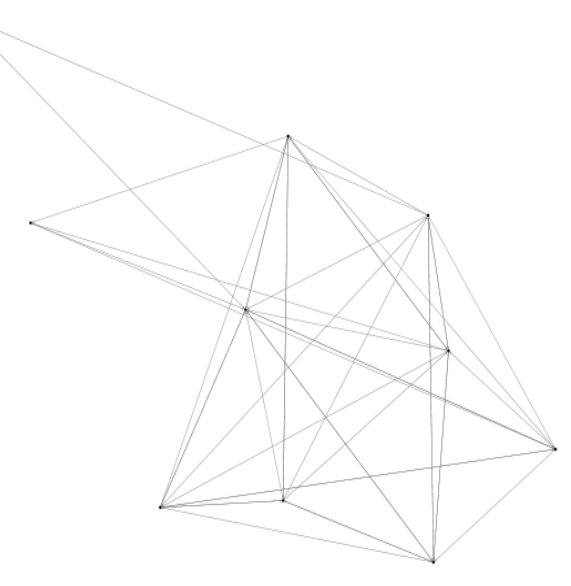

Figura 5. Matriz Adjacente e Grafo Log SisColab2014.2

Ao aplicar o algoritmo com a entrada deste log, obtemos:

1. A matriz de adjacência ponderada A de $\mathrm{G}$ está representada na Figura 5.

2. O grau de entrada está representado na Figura 5.

3. Vetor T ordenado: $\{1,2,5,5,5,7,8,9,11,55\}$

4. Teste de Diagrama de Caixas em $\mathrm{T}$

○ Mediana $=6 ; \mathbf{Q 1}=5 ; \mathbf{Q 3}=8,75 ; \mathbf{I Q R}=3,75 ; \mathbf{Z}=5,625 ; \mathbf{Q 3}+\mathbf{Z}=14,375$

\section{Conversação centrada}

Mais uma vez, analisando os valores do grau de entrada na figura 5 é fácil visualizar a grande diferença entre o número de mensagens endereçadas ao professor Marcelo e o número de mensagens endereçadas aos próprios alunos. De fato, o 
algoritmo identifica o grau de entrada 55 do professor como acima do limite superior 14,375. Também em relação a esta instância, o algoritmo identifica que a conversa foi centrada no professor.

\section{3. $\log$ TIAE2000.1}

Este log de bate-papo ocorreu no ambiente AulaNet (Fuks, 2000), na disciplina Tecnologia de Informação Aplicada à Educação (Lucena et al., 2000). A sessão foi realizada com nove participantes sendo oito alunos e um professor identificado pelo pseudônimo Humberto. Nesta sessão, o aluno com o pseudônimo Geraldo desempenhou o papel de moderador do debate. A Figura 6 mostra a matriz adjacente e o grafo correspondente a este $\log$.

\begin{tabular}{|c|c|c|c|c|c|c|c|c|c|}
\hline & 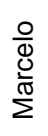 & $\begin{array}{l}\frac{0}{0} \\
\frac{0}{\frac{0}{0}} \\
\stackrel{ }{\amalg}\end{array}$ & 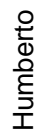 & $\frac{0}{\frac{0}{0}}$ & 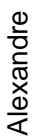 & $\underset{\frac{0}{\frac{\sigma}{\sigma}}}{\frac{0}{\Delta}}$ & 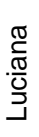 & 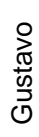 & $\frac{\frac{0}{0}}{\frac{0}{0}}$ \\
\hline Marcelo & 8 & 1 & 19 & 13 & 1 & 6 & 0 & 2 & 0 \\
\hline Fabricio & 2 & 1 & 4 & 2 & 0 & 0 & 0 & 0 & 0 \\
\hline Humberto & 21 & 2 & 21 & 12 & 4 & 6 & 5 & 4 & 3 \\
\hline Pablo & 12 & 3 & 15 & 33 & 0 & 4 & 0 & 2 & 0 \\
\hline Alexandre & 1 & 0 & 6 & 1 & 1 & 1 & 0 & 0 & 0 \\
\hline Liane & 8 & 0 & 8 & 5 & 1 & 1 & 0 & 0 & 0 \\
\hline Luciana & 0 & 0 & 8 & 4 & 0 & 1 & 0 & 0 & 0 \\
\hline Gustavo & 1 & 0 & 4 & 6 & 0 & 0 & 0 & 2 & 0 \\
\hline Geraldo & 0 & 0 & 3 & 3 & 0 & 0 & 0 & 0 & 1 \\
\hline $\begin{array}{l}\text { Grau de } \\
\text { entrada }\end{array}$ & 53 & 7 & 88 & 79 & 7 & 19 & 5 & 10 & 4 \\
\hline
\end{tabular}

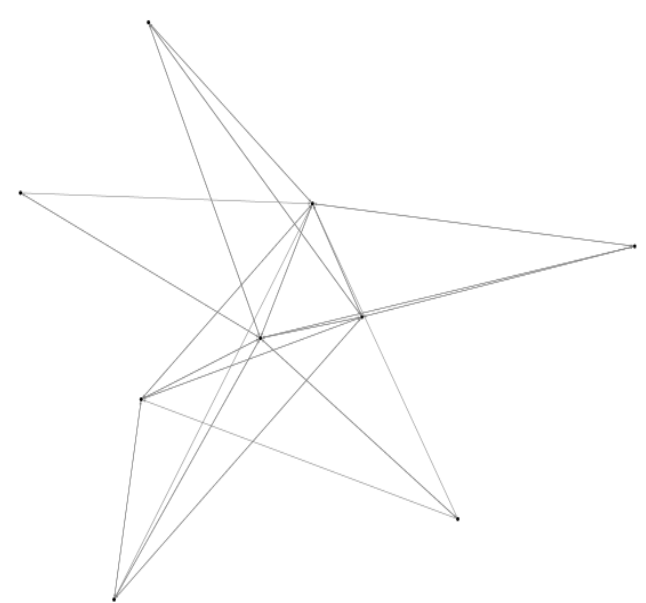

Figura 6. Matriz Adjacente e Grafo Log TIAE2000.1

Considerando agora a entrada deste $\log$, encontramos os seguintes resultados:

1. A matriz de adjacência ponderada A de $\mathrm{G}$ está representada na Figura 6.

2. O grau de entrada está representado na Figura 6.

3. Vetor T ordenado: $\{4,5,7,7,10,19,53,79,88\}$

4. Teste de Diagrama de Caixas em $\mathrm{T}$

○ Mediana=10; $\mathbf{Q 1 = 7 ; ~ Q 3 = 5 3 ; ~ I Q R = 4 6 ; ~ Z = 6 9 ; ~ Q 3 ~ + ~ Z ~ = 1 2 2 ~}$

\section{Conversação não centrada}

Aplicando o algoritmo proposto não foi identificado nenhum valor acima do limite superior 122. Assim, temos como saída do algoritmo a resposta que a conversação não foi centrada. Portanto, a conversação nesta sessão de bate-papo foi colaborativa.

\section{Conclusão}

Dado o novo cenário educacional, em que a EAD cresce cada vez mais no Brasil e que os professores-mediadores deveriam promover aulas baseadas na interação e colaboração entre os estudantes, é importante avaliarmos como tem ocorrido a conversação online. Neste artigo, apresentamos um método para analisar a conversação que ocorre no bate-papo. O método apresentou-se adequado para identificar se a conversação foi centrada no professor ou se foi estabelecida a colaboração entre os alunos. Como trabalho futuro, deseja-se adaptar o método para possibilitar a mesma análise em outros meios conversacionais, especialmente em fóruns de discussão (seja do Moodle, dos grupos do Facebook ou de outros sistemas). 


\section{Referências}

Calvão, L. D., Pimentel, M., Fuks, H. Do Email ao Facebook: Uma perspectiva evolucionista sobre os meios de conversação da internet. Rio de Janeiro: UNIRIO, 2014.

CensoEAD. <http://www.abed.org.br/censoead/censoEAD.BR_2012_pt.pdf> Acesso em: 23 out 2014.

Estruc, M., Pimentel, M. (2012). Portal Tagarelas: bate-papo para educação. XXIII Simpósio Brasileiro de Informática na Educação - SBIE. Rio de Janeiro, RJ.

Freeman, L. C. (2004) The Development of Social Network Analysis. A Study in the Sociology of Science. Empirical Press: Vancouver, 2004.

Fuks, H. Aprendizagem e Trabalho cooperativo no Ambiente AulaNet. Revista Brasileira de Informática na Educação, n 6. abril de 2000. Disponível na Internet via Web: http://anauel.cead.puc-rio.br/aulanet/index.html

Inep (2012) Censo da Educação Superior 2012. MEC, 2012. Disponível em <http://portal.mec.gov.br/index.php?option=com_docman\&task=doc_download\&gid $=9332 \&$ Itemid $=>$. Acessadoem 20 nov 2014 .

Löber, A., Schwabe, G., Grimmi S (2007). Audio vs. chat: The effects of group size on media choice. Proceedings of the $40^{\text {th }}$ HICCS Hawaii International Conference on System Sciences.

Lucena, C. J. P., Fuks, H., et. al. Tecnologia de Informação Aplicada a Educação: Um Meta Curso no Ambiente AulaNet [online]. Monografia em Ciência da Computação, Departamento de Informática, PUC-Rio. Rio de Janeiro, março de 2000. Disponível em: 〈http://anauel.cead.pucrio.br/aulanet/index.html>

Moraes, E. L. C. (2011) "Debatepapo: sequências conversacionais e visualização do cotexto para compreensão da conversação em bate-papo". 86 páginas. Dissertação de Mestrado. Departamento de Informática Aplicada, UNIRIO.

Netto, A.T.C. (2014) Sugestão de Associações entre Mensagens de Bate-papo: Um experimento com o sistema debatepapo V.2. Dissertação de Mestrado em Informática, UNIRIO, Rio de Janeiro, Brasil.

Pimentel, M.G., Fuks, H. e Lucena, C.J.P. (2003) "Debati, debati... aprendi? Investigações sobre o papel educacional das ferramentas de bate-papo", IX Workshop sobre Informática na Escola - WIE2003, Agosto.

Piva, D.J., Pupo, R., Gamez, L., Oliveira, S. (2011). EAD na Prática - Planejamento, métodos e ambientes de educação online. Elsevier Editora Ltda., 2011, v.,p 164-170.

Recuero, R. (2012) A conversação em rede: comunicação mediada pelo computador e redes sociais na Internet - Porto Alegre: Ed. Sulina.

Rocha, E.B., Pimentel, M., Diniz, M.C. Quantidade de Participantes em Bate-papo Educacional: um Modelo Baseado em Teoria de Filas. Revista Brasileira de Informática na Educação (no prelo). 2015.

Silva, M. (2010) Avaliação da Aprendizagem em Educação Online. 2010. Entrevista publicada no Youtube<http://youtu.be/S7uUd6afEYE>, acessado em 18 abr 2015. 
CBIE-LACLO 2015

Anais do XXVI Simpósio Brasileiro de Informática na Educação (SBIE 2015)

Silva, M. (2010b) Educar na Cibercultura: Desafios à formação de professores para docência em cursos online. Jan-Jun 2010.

Singla, P., Richardson, M. Yes, there is a correlation:-from social networks to personal behavior on the web. In Proceeding of the 17th international conference on World Wide Web , pages 655-664. ACM, 2008

Stahl, G. (Ed.). (2009). Studying virtual math teams. New York, NY: Springer. 